\title{
Metal Allergy and Systemic Contact Dermatitis: An Overview
}

\author{
Yoko Yoshihisa and Tadamichi Shimizu \\ Department of Dermatology, Graduate School of Medicine and Pharmaceutical Sciences, University of Toyama, \\ Toyama 930-0194, Japan \\ Correspondence should be addressed to Tadamichi Shimizu, shimizut@med.u-toyama.ac.jp
}

Received 8 March 2012; Accepted 6 April 2012

Academic Editor: Alex Zvulunov

Copyright ( $) 2012$ Y. Yoshihisa and T. Shimizu. This is an open access article distributed under the Creative Commons Attribution License, which permits unrestricted use, distribution, and reproduction in any medium, provided the original work is properly cited.

\begin{abstract}
Contact dermatitis is produced by external skin exposure to an allergen, but sometimes a systemically administered allergen may reach the skin and remain concentrated there with the aid of the circulatory system, leading to the production of systemic contact dermatitis (SCD). Metals such as nickel, cobalt, chromium, and zinc are ubiquitous in our environment. Metal allergy may result in allergic contact dermatitis and also SCD. Systemic reactions, such as hand dermatitis or generalized eczematous reactions, can occur due to dietary nickel or cobalt ingestion. Zinc-containing dental fillings can induce oral lichen planus, palmoplantar pustulosis, and maculopapular rash. A diagnosis of sensitivity to metal is established by epicutaneous patch testing and oral metal challenge with metals such as nickel, cobalt, chromium, and zinc. In vitro tests, such as the lymphocyte stimulating test (LST), have some advantages over patch testing to diagnose allergic contact dermatitis. Additionally, the determination of the production of several cytokines by primary peripheral blood mononuclear cell cultures is a potentially promising in vitro method for the discrimination of metal allergies, including SCD, as compared with the LST.
\end{abstract}

\section{Introduction}

Contact dermatitis is usually produced by external exposure of the skin to an allergen; however, sometimes a systemically administered allergen may reach the skin through the circulatory system and thereby produce systemic contact dermatitis. Systemic contact dermatitis (SCD) is an inflammatory skin disease that is known to occur with exposure to drugs, foods, and dental metals. A variety of types of skin eruptions have been reported, including flares of previous patch test sites, symmetrical intertriginous and flexural exanthema, exfoliative erythroderma, and widespread dermatitis [1].

Metals such as nickel, cobalt, chromium, and zinc are ubiquitous in our environment. During the 20th century, industrialization and modern living resulted in increased cutaneous exposure to these metals and hence an increased incidence of metal allergies [2]. Metal allergies may result in allergic contact dermatitis. Metals that are electrophilic have the ability to ionize and react with proteins, thus forming complexes that can be recognized by dendritic cells, which allows for sensitization to occur [3]. Cases of contact dermatitis caused by cutaneous exposure to cosmetics products and jewelry that contain nickel have been reported in the literature. The thinness of the stratum corneum and intermittent exposure to sweat on the eyelids have been associated with increased nickel absorption through the skin from cosmetics, allowing lower nickel concentrations to elicit a reaction [4]. Cobalt is a strong skin sensitizer [5]. Over the years, occupational exposure to cobalt has been primarily observed in metal workers, bricklayers, and pottery workers. Contact dermatitis that results from direct contact to an allergen is the most common and easiest form of metal allergy to identify. However, the timely recognition of the type of systemic skin inflammation known as SCD and its varying presentations is critical as it can result in more chronic and severe symptoms.

\section{Metals and SCD}

2.1. Nickel and SCD. Nickel is a chemical element found ubiquitously in the environment and is used with a high frequency worldwide. This metal is manufactured into steel and a variety of alloys containing cobalt, palladium, iron, titanium, gold, and magnesium [6]. Sensitized individuals generally experience a predictable localized response 
following cutaneous exposure to nickel, including erythema, vesicle formation, scaling, and pruritus. According to recent studies, females have an about 4-fold higher relative risk of developing allergic contact dermatitis to nickel compared with males [6].

Systemic reactions, such as generalized eczematous reactions or dyshidrotic hand eczema, can occur due to dietary ingestion of nickel. In 1984, Andersen et al. coined the term "baboon syndrome" to describe the generalized dermatitis of the buttocks, anogenital area, flexures, and eyelids that is frequently observed in patients with SCD to nickel [7]. Nickel is present in most dietary items, and food is considered to be a major source of exposure to nickel for the general population. Certain foods are routinely found to be high in nickel content. Nickel present in the diet of a nickelsensitive person can provoke SCD. For example, SCD can be elicited in nickel-sensitive individuals from the consumption of foods with a high nickel content, such as cocoa [8]. In such patients, adherence to a low-nickel diet and avoidance of local exposure to metal objects result in the disappearance of skin symptoms. Silvestri and Barmettler reported the case of a nickel-sensitive patient with a 1.5 year history of treatmentresistant pruritus ani [9]. The patient disclosed a habit of daily peanut butter consumption. His symptoms resolved with a restriction of dietary nickel [9]. A study of systemic nickel allergy found a dose-response relationship between nickel ingestion and the occurrence of dermatitis flare-ups [10]. Of note, for most nickel-allergic patients, a single dose of $4 \mathrm{mg}$ of nickel will result in widespread dermatitis [10]. It is recommend that individuals with food-related flare-ups of nickel dermatitis consume a low-nickel diet [11].

Nickel is considered to be the most frequent contact allergen for patients with $\mathrm{AD}$ [12]. A recently published study of a German population showed a positive association between filaggrin mutations, which have been shown to be strongly associated with $\mathrm{AD}$, and contact sensitization to nickel [13]. Another study also reported a positive association between nickel sensitization and $\mathrm{AD}$, in a subanalysis of nonpierced women [14].

It is necessary to be aware of the systemic reactions that occur with SCD, which can be chronic and can produce severe symptoms that may often be mistaken for $\mathrm{AD}$ [15]. Initially, Shanon reported that patients with SCD occasionally experience a skin manifestation similar to $\mathrm{AD}$ called "pseudoatopic dermatitis" [16]. Hsu et al. recently reported four cases of children with variable presentations of SCD to nickel [15]. For each of these patients, the presence of clinically relevant exposure to nickel was confirmed with dimethylglyoxime testing. One of these patients, 16 years old, had a nine-year history of pruritic dermatitis that began on her infraumbilical area and arms. During the past year, the dermatitis had spread to the remainder of her body, including her face, and the patient was thus believed to have $\mathrm{AD}[15]$.

2.2. Cobalt and SCD. Although nickel sensitivity is more common than cobalt sensitivity, the two are frequently linked. Rystedt and Fischer reported that a quarter of nickelsensitive patients developed a cobalt allergy and patients with simultaneous nickel and cobalt allergies have more severe dyshidrotic eczema [17]. It was proposed that a lowcobalt-diet reduced the dyshidrotic eczema flares in cobalt allergic patients [18]. Therefore, the ingestion of increased amounts of cobalt through food should be added to the list of triggering factors for SCD.

Furthermore, cobalt is contained in a variety of materials. Hard metal is manufactured by means of a powder metallurgical process in which about $90 \%$ tungsten carbide, small amounts of other metal carbides, and polyethylene glycol are mixed with about $10 \%$ metallic cobalt, which is used as a binding agent. Hard metal manufacturing involves pressing, forming, sintering, grinding, and etching or color marking. Cobalt exposure via inhalation may lead to cobaltrelated asthma. Hard metal workers may develop cough, wheezing, and dyspnea that often improve during weekends and holidays [18]. The occurrence of localized contact dermatitis due to occupational exposure to cobalt in the hard metal industry has also been reported $[19,20]$.

However, contact with a hard metal powder in the workplace is a rare cause of SCD. In particular, there has been only one report of occupational cobalt-induced SCD [21]. The case was a 19-year-old male who had worked as a grinder for 1 month in a hard metal factory. The hard metals used in the factory contained cobalt. The patient developed erythema on his hands 2 weeks after starting the work, which thereafter progressed to a generalized eczematous eruption with itching [21]. Patch testing showed positive reactions for $1 \%$ cobalt chloride. After changing his workplace, his skin rush disappeared. In this case, the recurrent eczematous lesions of the hands were associated with a flare of systemic dermatitis and were highly suggestive of SCD triggered by cobalt inhalation. Dermatologists should, therefore, remind such patients to pay increased attention to avoid all kinds of cobalt exposure in their daily life and work.

2.3. Chromium and SCD. The element chromium was discovered by Vaquelin in 1798. It is ubiquitous in the environment and is widely used in the plating, leather tanning, pigmentation, dye production, metallurgy, and chemical industries and is found in cement as a byproduct of the cement manufacturing process itself $[22,23]$. When exposed to skin, chromium salts can induce cutaneous irritation, which may progress to SCD in cases of chromium hypersensitivity [24]. Chromate-induced SCD is primarily exacerbated by skin contact with hexavalent and trivalent chromium compounds [25]; however, the ingestion of the allergen in the dichromate form has also been reported to cause exacerbations [26-29]. The oral ingestion of trivalent chromium, that is, chromium picolinate, for nutritional supplementation has been reported to cause SCD [30]. Recently, SCD resulting from the ingestion of chromium chloride in a multivitamin/multimineral tablet has been reported [31].

Metal allergy has also been associated with device failures following the insertion of intracoronary stents, hip and knee prostheses, and other implants. Gao et al. reported a case of SCD most likely caused by exposure to chromium after 


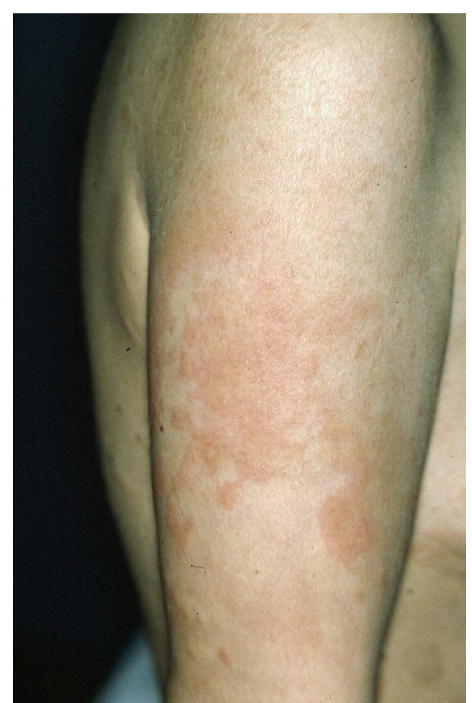

(a)

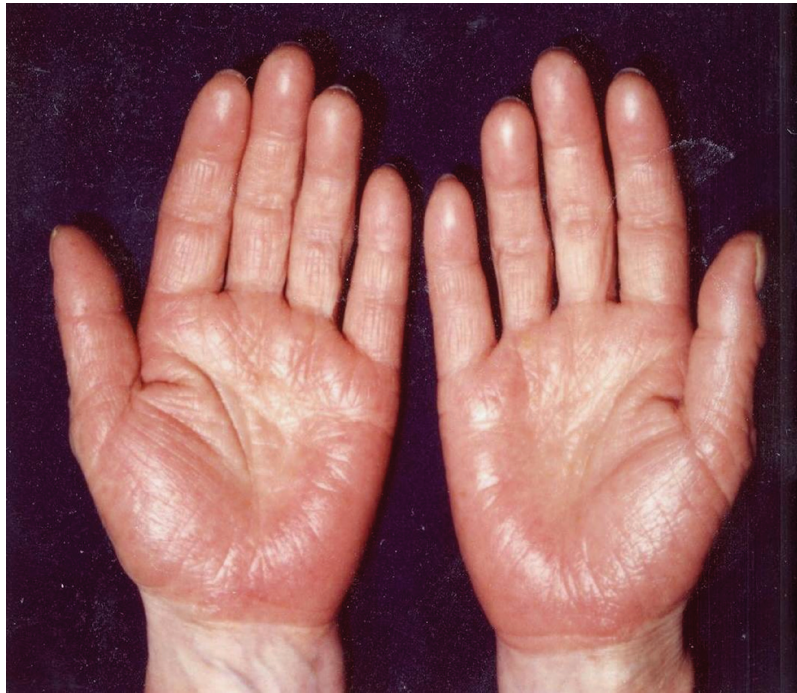

(b)

Figure 1: (a) A 49-year-old Japanese female with a diffuse edematous erythema with papules over her entire body. (b) The oral challenge test with zinc sulfate caused exacerbation of the preexisting eruptions on her palms, including itching edematous erythema.

a total knee arthroplasty, although this complication is very rare [32]. The majority of total joint prostheses are now made of cobalt-chromium alloys with a nickel content of less than $1 \%$ [33]. The occurrence of SCD is particularly uncommon following total knee arthroplasty because there is a polyethylene insert between the femoral and tibial components and no metal-on-metal contact exists.

2.4. Zinc and SCD. Zinc is an essential trace element involved in many physiological functions, including catalytic and structural roles in metalloenzymes, as well as regulatory roles in diverse cellular processes, such as synaptic signaling and gene expression. Zinc is widely used in dental restoration. The previously reported dental metal eruptions caused by zinc have included oral lichen planus [34], palmoplantar pustulosis [35], and a maculopapular rash [36]. It has also been reported to cause severe symptoms in cases of SCD. One case was a 49-year-old Japanese female who developed facial edema, blepharedema, and pruritic edematous erythema with papules over her entire body. Based on the results of a metal patch test, lymphocyte stimulating test (LST), and zinc challenge test, a diagnosis of zinc-allergic SCD was made (Figure 1) [37]. This case had four teeth that had been treated with metal fillings, which likely contained zinc. All of the patient's dental fillings were completely removed, and her diet was changed to a zinc-restricted diet. Two weeks later, the majority of the skin lesions, which had lasted for four months, subsided rapidly [37].

Saito et al. reported another severe case of SCD that developed because of the zinc contained in dental fillings, in which generalized flare-up reactions occurred from a zinc patch test [38]. In this case, one may suspect the amount of zinc that can be absorbed through the skin or oral mucosa compared with that obtained through dietary zinc intake to be small.

\section{Diagnosis of Metal Sensitivity}

Epicutaneous path testing has been used to diagnose metal sensitivity. It is the primary tool to diagnose allergens that cause allergic contact dermatitis. The main advantages of patch tests are that they can be completed without hospital surveillance since they rarely induce adverse reactions. Therefore, a patch test evaluation is the gold standard for detecting metal hypersensitivity. However, the accuracy of this method is strongly dependent on the experience of the observer, and distinguishing doubtful-positive from positive patch test reactions for different reagents remains difficult. Sometimes false-positive and negative reactions are observed in conditions of existing dermatitis. Some patch test substances, such as cobalt, nickel, copper, and chromium, sometimes cause false-positives and pustule formation [39, 40].

Oral metal challenges with nickel, cobalt, chrome, and zinc are sometimes performed and are diagnostic for metal allergies, especially SCD. However, flare-up reactions sometimes appear at previous sites of eczema, including hand eczema, and at patch test sites after an oral challenge [41].

In vitro tests, such as the LST, have some advantages over patch testing to diagnose allergic contact dermatitis. First, the LST does not cause flare-ups or exacerbation of symptoms in patients, is objective, and can be used in clinical situations where patch testing is not recommended [42]. However, the LST has not yet been sufficiently optimized or validated to be used as the sole routine diagnostic method for confirming a suspicion of a contact allergy. With regard to the diagnosis of nickel allergy, the task is made quite difficult because of the frequent overlap in test results between nickelallergic and nonallergic subjects, which may be partly due to a nonspecific, mitogenic effect exerted by nickel [43].

It is useful to assess metal-induced cytokine profiles using the in vitro stimulation of primary peripheral blood 
mononuclear cells (PBMCs) with metal salts alone. Stimulation with nickel, cobalt, and chromium leads to a specific pattern of cytokine secretion in PBMC cultures obtained from metal-allergic patients, which involves both Th1- and Th2-type cytokines [44-47]. Based on a blood analysis of 14 patients with SCD to nickel, IFN- $\gamma$ and IL-5 seem to play an important role in the pathogenesis of SCD [48]. Studies of the relationship between zinc and cytokines showed that zinc increased monokine secretion more efficiently than other related divalent cations, including cobalt, nickel, and mercury [49]. Furthermore, zinc stimulation of the PBMCs obtained from SCD patients showed higher macrophage migration inhibitory factor (MIF) and TNF- $\alpha$ secretion compared to that found in healthy subjects [37]. MIF increases TNF- $\alpha$ production and is thought to play an important role in contact hypersensitivity responses [50]. MIF is secreted from both Th1- and Th2-type cells [51]. This suggests that the presence of zinc in the peripheral blood of zinc-allergic patients induces PBMCs to produce increased levels of MIF, which could lead to SCD.

In conclusion, the determination of Th1- and Th2-type cytokine production in PBMC cultures is a potentially promising in vitro method for diagnosing metal allergies, including SCD. Therefore, the analysis of PBMC cultures may be helpful in confirming the diagnosis of SCD caused by metal allergy in patients with positive patch testing.

\section{Acknowledgment}

This research was supported by a Grant-in-Aid for research (no. 20591337) from the Ministry of Education, Science and Culture of Japan.

\section{References}

[1] R. I. Nijhawan, M. Molenda, M. J. Zirwas, and S. E. Jacob, "Systemic contact dermatitis," Dermatologic Clinics, vol. 27, no. 3, pp. 355-364, 2009.

[2] J. P. Thyssen, A. Linneberg, T. Menné, and J. D. Johansen, “The epidemiology of contact allergy in the general populationprevalence and main findings," Contact Dermatitis, vol. 57, no. 5, pp. 287-299, 2007.

[3] S. E. Jacob and T. Zapolanski, "Systemic contact dermatitis," Dermatitis, vol. 19, no. 1, pp. 9-15, 2008.

[4] J. P. Thyssen, A. Linneberg, T. Menné, N. H. Nielsen, and J. D. Johansen, "No association between nickel allergy and reporting cosmetic dermatitis from mascara or eye shadow: a cross-sectional general population study," Journal of the European Academy of Dermatology and Venereology, vol. 24, no. 6, pp. 722-725, 2010.

[5] J. E. Wahlberg and A. Boman, "Sensitization and testing of guinea pigs with cobalt chloride," Contact Dermatitis, vol. 4, no. 3, pp. 128-132, 1978.

[6] L. K. Lu, E. M. Warshaw, and C. A. Dunnick, "Prevention of Nickel allergy: the case for regulation?" Dermatologic Clinics, vol. 27, no. 2, pp. 155-161, 2009.

[7] K. E. Andersen, N. Hjorth, and T. Menne, "The baboon syndrome: systematically-induced allergic contact dermatitis," Contact Dermatitis, vol. 10, no. 2, pp. 97-100, 1984.

[8] B. Krecisz, D. Chomiczewska, M. Kiec-Swierczynska, and A. Kaszuba, "Systemic contact dermatitis to nickel present in cocoa in 14-year-old boy," Pediatric Dermatology, vol. 28, no. 3, pp. 335-336, 2011.

[9] D. L. Silvestri and S. Barmettler, "Pruritus ani as a manifestation of systemic contact dermatitis: resolution with dietary nickel restriction," Dermatitis, vol. 22, no. 1, pp. 50-55, 2011.

[10] C. S. Jensen, T. Menné, S. Lisby, J. Kristiansen, and N. K. Veien, "Experimental systemic contact dermatitis from nickel: a dose-response study," Contact Dermatitis, vol. 49, no. 3, pp. 124-132, 2003.

[11] A. Sharma, "Relationship between nickel allergy and diet," Indian Journal of Dermatology, Venereology and Leprology, vol. 73, no. 5, pp. 307-312, 2007.

[12] F. Giordano-Labadie, F. Rancé, F. Pellegrin, J. Bazex, G. Dutau, and H. P. Schwarze, "Frequency of contact allergy in children with atopic dermatitis: results of a prospective study of 137 cases," Contact Dermatitis, vol. 40, no. 4, pp. 192-195, 1999.

[13] N. Novak, H. Baurecht, T. Schäfer et al., "Loss-of-function mutations in the Filaggrin gene and allergic contact sensitization to nickel," Journal of Investigative Dermatology, vol. 128, no. 6, pp. 1430-1435, 2008.

[14] J. P. Thyssen, A. Linneberg, K. Engkilde et al., "Contact sensitization to common haptens is associated with atopic dermatitis:new insight," British Journal of Dermatology. In press.

[15] J. W. Hsu, C. Matiz, and S. E. Jacob, "Nickel allergy: localized, Id, and systemic manifestations in children," Pediatric Dermatology, vol. 28, no. 3, pp. 276-280, 2011.

[16] J. Shanon, "Pseudo-atopic dermatitis. Contact dermatitis due to chrome sensitivity simulating atopic dermatitis.," Dermatologica, vol. 131, no. 3, pp. 176-190, 1965.

[17] I. Rystedt and T. Fischer, "Relationship between nickel and cobalt sensitization in hard metal workers," Contact Dermatitis, vol. 9, no. 3, pp. 195-200, 1983.

[18] J. Stuckert and S. Nedorost, "Low-cobalt diet for dyshidrotic eczema patients," Contact Dermatitis, vol. 59, no. 6, pp. 361365, 2008.

[19] L. Schwartz, S. M. Peck, K. E. Blair, and K. E. Markuson, "Allergic dermatitis due to metallic cobalt," Journal of Allergy, vol. 16, no. 1, pp. 51-53, 1945.

[20] E. SKOG, "Skin affections caused by hard metal dust.," Industrial Medicine \& Surgery, vol. 32, pp. 266-268, 1963.

[21] Y. Asano, T. Makino, O. Norisugi, and T. Shimizu, "Occupational cobalt induced systemic contact dermatitis," European Journal of Dermatology, vol. 19, no. 2, pp. 166-168, 2009.

[22] M. Costa and C. B. Klein, "Toxicity and carcinogenicity of chromium compounds in humans," Critical Reviews in Toxicology, vol. 36, no. 2, pp. 155-163, 2006.

[23] D. Burrows, "Adverse chromate reactions on the skin," in Chromium: Metabolism and Toxicity, D. Burrows, Ed., pp. 137163, CRC Press, Boca Raton, Fla, USA, 2000.

[24] J. G. Marks, P. Elsner, and V. De Leo, Contact and Occupational Dermatology, Mosby, Mo, USA, 2002.

[25] M. B. Hansen, J. D. Johansen, and T. Menné, "Chromium allergy: significance of both $\mathrm{Cr}(\mathrm{III})$ and $\mathrm{Cr}(\mathrm{VI})$," Contact Dermatitis, vol. 49, no. 4, pp. 206-212, 2003.

[26] K. Kaaber and N. K. Veien, "The significance of chromate ingestion in patients allergic to chromate," Acta DermatoVenereologica, vol. 57, no. 4, pp. 321-323, 1977.

[27] J. Van Ulsen, E. Stolz, and Van Joost Th., "Chromate dermatitis from a homeopathic drug," Contact Dermatitis, vol. 18, no. 1, pp. 56-57, 1988.

[28] T. Menné, N. Veien, K. E. Sjølin, and H. I. Maibach, "Systemic contact dermatitis," American Journal of Contact Dermatitis, vol. 5, no. 1, pp. 1-12, 1994. 
[29] N. K. Veien, T. Hattel, and G. Laurberg, "Chromate-allergic patients challenged orally with potassium dichromate," Contact Dermatitis, vol. 31, no. 3, pp. 137-139, 1994.

[30] J. F. Fowler Jr., "Systemic contact dermatitis caused by oral chromium picolinate," Cutis, vol. 65, no. 2, p. 116, 2000.

[31] E. Özkaya, Z. Topkarci, and G. Özarmaǧan, "Systemic allergic dermatitis from chromium in a multivitamin/multimineral tablet," Contact Dermatitis, vol. 62, no. 3, p. 184, 2010.

[32] X. Gao, R. X. He, S. G. Yan, and L. D. Wu, "Dermatitis Associated With Chromium Following Total Knee Arthroplasty," Journal of Arthroplasty, vol. 26, no. 4, pp. 665.e13-665.e16, 2011.

[33] A. Afolaranmi, J. Tettey, R.M. Meek et al., "Release of chromium from orthopaedic arthroplasties," The Open Orthopaedics Journal, vol. 2, pp. 10-18, 2008.

[34] T. Ido, M. Kumakiri, T. Kiyohara, T. Sawai, and Y. Hasegawa, "Oral lichen planus due to zinc in dental restorations," Contact Dermatitis, vol. 47, no. 1, p. 51, 2002.

[35] T. Yanagi, T. Shimizu, R. Abe, and H. Shimizu, "Zinc dental fillings and palmoplantar pustulosis," Lancet, vol. 366, no. 9490, p. 1050, 2005.

[36] T. Shimizu, S. Kobayashi, and M. Tanaka, "Systemic contact dermatitis to zinc in dental fillings," Clinical and Experimental Dermatology, vol. 28, no. 6, pp. 675-676, 2003.

[37] T. Yanagi, K. Kodama, Y. Yoshihisa, H. Shimizu, and T. Shimizu, "Macrophage migration inhibitory factor in zincallergic systemic contact dermatitis," Cytokine, vol. 35, no. 5-6, pp. 270-274, 2006.

[38] N. Saito, N. Yamane, W. Matsumura et al., "Generalized exacerbation of systemic allergic dermatitis due to zinc patch test and dental treatments," Contact Dermatitis, vol. 62, no. 6, pp. 372-373, 2010.

[39] N. Hjorth, "Diagnostic patch testing," in Dermatoxicology and Pharmacology, F. Marzulli and H. I. Maibach, Eds., p. 344, John Wiley and Sons, New York, NY, USA, 1977.

[40] T. Fischer and I. Rystedt, "False-positive, follicular and irritant patch test reactions to metal salts," Contact Dermatitis, vol. 12, no. 2, pp. 93-98, 1985.

[41] M. Hindsén, M. Bruze, and O. B. Christensen, "Flare-up reactions after oral challenge with nickel in relation to challenge dose and intensity and time of previous patch test reactions," Journal of the American Academy of Dermatology, vol. 44, no. 4, pp. 616-623, 2001.

[42] C. S. Jensen, S. Lisby, J. K. Larsen, N. K. Veien, and T. Menné, "Characterization of lymphocyte subpopulations and cytokine profiles in peripheral blood of nickel-sensitive individuals with systemic contact dermatitis after oral nickel exposure," Contact Dermatitis, vol. 50, no. 1, pp. 31-38, 2004.

[43] S. Silvennoinen-Kassinen, "The specificity of a nickel sulphate reaction in vitro: a family study and a study of chromiumallergic subjects," Scandinavian Journal of Immunology, vol. 13, no. 3, pp. 231-235, 1981.

[44] L. Borg, J. M. Christensen, J. Kristiansen, N. H. Nielsen, T. Menné, and L. K. Poulsen, "Nickel-induced cytokine production from mononuclear cells in nickel-sensitive individuals and controls. Cytokine profiles in nickel-sensitive individuals with nickel allergy-related hand eczema before and after nickel challenge," Archives of Dermatological Research, vol. 292, no. 6, pp. 285-291, 2000.

[45] H. Falsafi-Amin, L. Lundeberg, M. Bakhiet, and K. Nordlind, "Early DNA synthesis and cytokine expression in the nickel activation of peripheral blood mononuclear cells in nickelallergic subjects," International Archives of Allergy and Immunology, vol. 123, no. 2, pp. 170-176, 2000.
[46] E. Jakobson, K. Masjedi, N. Ahlborg, L. Lundeberg, A. T. Karlberg, and A. Scheynius, "Cytokine production in nickelsensitized individuals analysed with enzyme-linked immunospot assay: possible implication for diagnosis," British Journal of Dermatology, vol. 147, no. 3, pp. 442-449, 2002.

[47] J. T. Minang, I. Areström, M. Troye-Blomberg, L. Lundeberg, and N. Ahlborg, "Nickel, cobalt, chromium, palladium and gold induce a mixed Th1- and Th2-type cytokine response in vitro in subjects with contact allergy to the respective metals," Clinical and Experimental Immunology, vol. 146, no. 3, pp. 417-426, 2006.

[48] E. Czarnobilska, P. Thor, J. Kaszuba-Zwoinska et al., "Response of peripheral blood mononuclear leukocyte to nickel stimulation in patients with systemic and contact allergy to nickel," Przegla, d Lekarski, vol. 63, no. 12, pp. 12761280, 2006.

[49] N. Wellinghausen, C. Driessen, and L. Rink, "Stimulation of human peripheral blood mononuclear cells by zinc and related cations," Cytokine, vol. 8, no. 10, pp. 767-771, 1996.

[50] T. Shimizu, R. Abe, J. Nishihira et al., "Impaired contact hypersensitivity in macrophage migration inhibitory factordeficient mice," European Journal of Immunology, vol. 33, no. 6, pp. 1478-1487, 2003.

[51] U. Schurigt, C. Pfirschke, I. M. Irmler et al., "Interactions of T helper cells with fibroblast-like synoviocytes: up-regulation of matrix metalloproteinases by macrophage migration inhibitory factor from both Th1 and Th2 cells," Arthritis and Rheumatism, vol. 58, no. 10, pp. 3030-3040, 2008. 


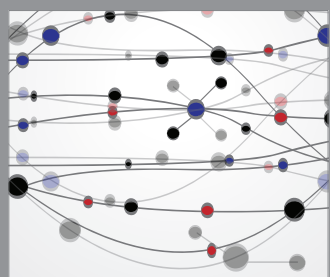

The Scientific World Journal
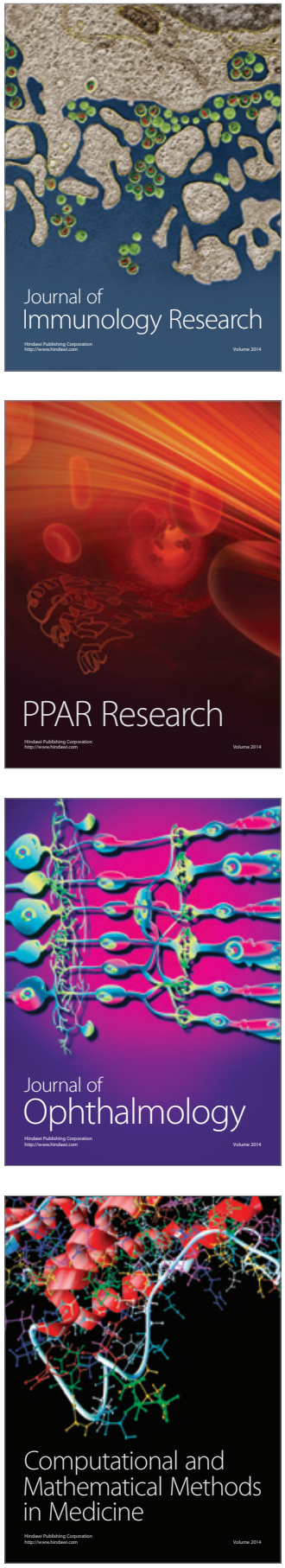

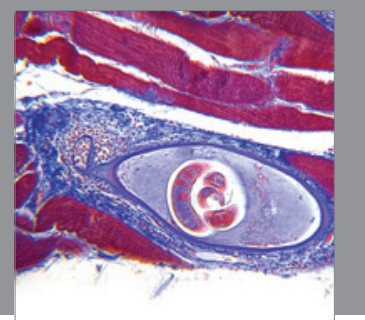

Gastroenterology

Research and Practice
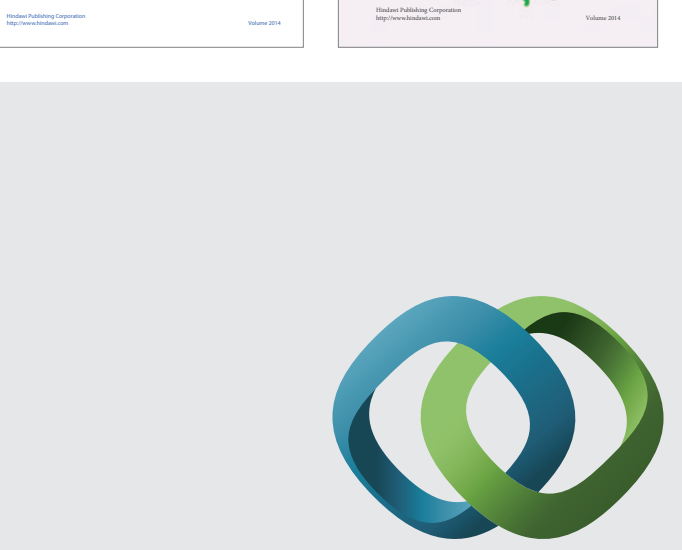

\section{Hindawi}

Submit your manuscripts at

http://www.hindawi.com
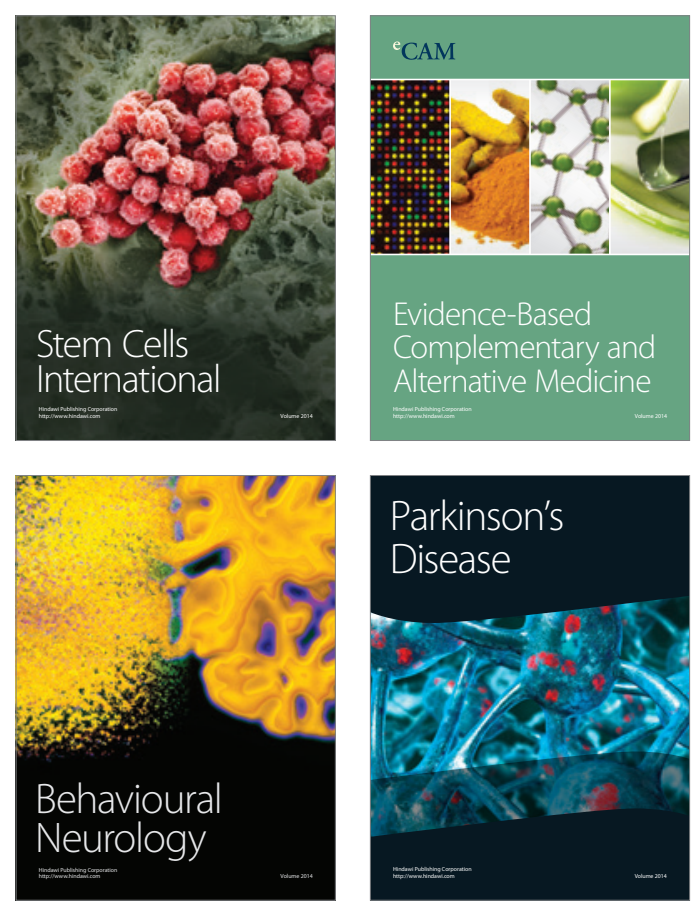

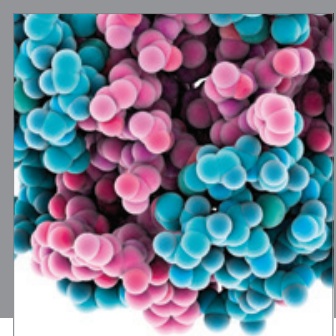

Journal of
Diabetes Research

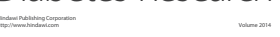

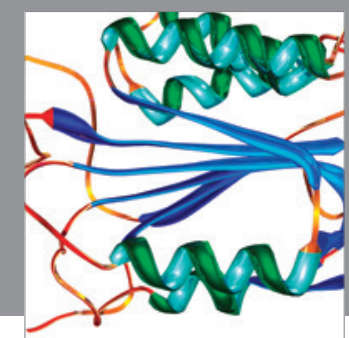

Disease Markers
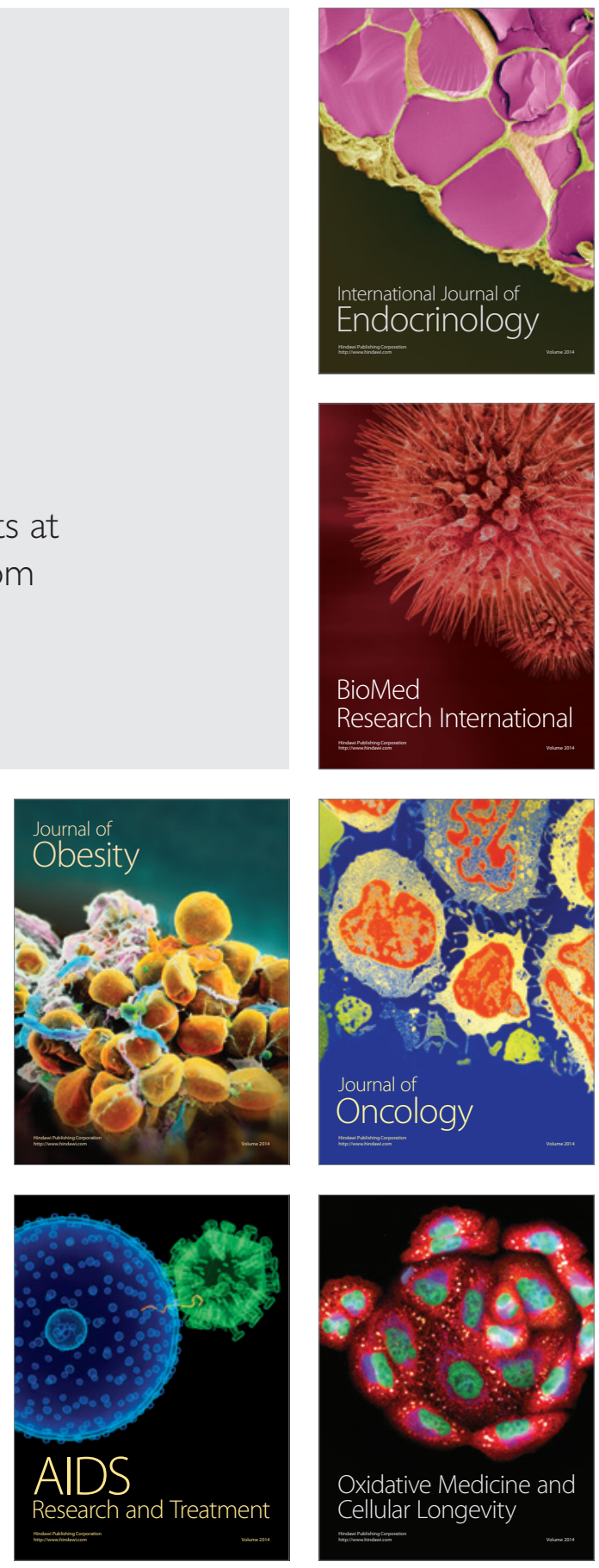